\section{Process and quality of peer review in scientific Nursing journals}

\section{Wai Tong Chien}

School of Nursing, Faculty of Health and Social Sciences, The Hong Kong

Polytechnic University, Hung Hom, Kowloon, Hong Kong S.A.R., P.R. China

\begin{abstract}
To be high quality and international recognized scientific journal for health care professions, such as medical and nursing disciplines, a well-structured and effective peer review system is of an utmost importance. Beckstead (2009) in his guest editorial published in the September issue of International Journal of Nursing Studies suggested that it was important for authors, as well as editors and publishers, to have a clear understanding of whom the intended readers of the journal are; and for the peer reviewers, to answer a key question: whom are we writing to? and more importantly, to assure a transparent, rigorous and quality peer-review. Their questions and declarations stimulated us to think about two important issues: first, the importance of a high quality and effective peer-review in a scientific nursing journal and second, the peer-review system, its monitoring and contribution to quality improvement.
\end{abstract}

\section{Introduction}

Scientific journals are periodical publications intended to report new research findings in order to enhance the progress of science. To be a high quality and international recognized scientific journal for health care professions such as medical and nursing disciplines, a well-structured and effective peer review system is of an utmost importance. The articles accepted for publication in the scientific journals should have been peer reviewed, in an attempt to ensure that articles meet the journal's standards of quality, and scientific validity. An independent, scholarly peer review or refereeing system should be set up, in which there is a process of subjecting an author's research report (often named an original article by the journals) to the scrutiny of others who are experts in the same field, before the report or manuscript is published in a scientific journal. Such peer review system requires a community of experts in a given (and specifically defined) field, who are qualified and able to perform impartial review. A fair and quality review, especially of work in less narrowly defined or within inter-disciplinary fields, may be difficult to be achieved; and the significance of an idea may never be widely appreciated among peer reviewers with different clinical or professional backgrounds. Although peer review is generally considered essential to academic quality, and used in most important scientific publications, it has been criticized as ineffective, slow and misunderstood due to various reasons such as difficulty in recruiting experienced reviewers with a variety of specialty areas and an editor's challenge and bias in soliciting reviews from a list of reviewers who may not be willing or time available for referee the article over that period. ${ }^{1}$

Peer reviewers should provide an objective critical evaluation of a manuscript and make a recommendation to the Editor-in-Chief by deciding whether it is well-written or needs a lot of work to make it acceptable. Some journals may also request the reviewers to indicate if the manuscript requires its English grammar, punctuation or spelling and referencing to be corrected. Beckstead ${ }^{2}$ in his guest editorial published in the September issue of International Journal of Nursing Studies suggested that it was important for reviewers, as well as editors and publishers, to understand an important issue when performing peer review of a manuscript: To whom are we writing? and more importantly, to insure a transparency, rigor and quality of peer reviews. The latter concern is also a crucial question suggested by the International Committee of Medical Journal Editors ${ }^{3}$ in their papers on the major considerations for peer review in disseminations and reporting of research projects. Their questions and declarations stimulated us to think about two important issues: first, the importance of high quality and effective peer reviews in an international scientific nursing journal; and second, a well-established peer review system and its monitoring and contribution to quality improvement.

\section{Purpose and quality of a peer-} reviewed scientific nursing journal

It has been argued that peer-reviewed scientific journals should provide a forum for the dissemination of research and scholarly ideas between scientists. I agree partially with this argument that an international peer-reviewed scientific nursing journal should provide a forum for publication of original papers or research reports of the highest standard. In addition to full research reports, high quality papers should be published to report and disseminate research evidences and dialogues, including literature reviews, discussion papers and commentaries, in order to enhance exchanges of new ideas and experiences on research topics among nurse researchers and
Correspondence: Wai Tong Chien,

School of Nursing, Faculty of Health and Social Sciences, The Hong Kong Polytechnic University Hung Hom, Kowloon, Hong Kong S.A.R., P.R. China.

Tel. +86852.2766 .5648 - Fax: +86.852 .23649663 . E-mail: hschien@inet.polyu.edu.hk;

Key words: peer review, nursing journals, readership.

Conflict of interest: the authors report no conflicts of interest.

This work is licensed under a Creative Commons Attribution NonCommercial 3.0 License (CC BYNC 3.0).

(C) Copyright W.T. Chien 2011

Licensee PAGEPress, Italy

Nursing Reports 2011; 1:e5

doi:10.4081/nursrep.2011.e5

thus contributing to the continuing development of nursing science. Adding to this general view, continuous infusion of such high quality new knowledge generated by nurse researchers is also a foundation for ongoing improvement in the provision of care to individuals, families, and the communities across borders and cultures. Readers of scholarly nursing journals are not only nurse academics or Ph.D. holders but also they may come from a wide field of international readers, comprising nurses, midwives, educators, policy holders and administrators, researchers, theorists, and philosophers in all the areas of health care sciences (i.e., similar to that stated in the aims of most nursing journals). Scientific peers, nurses in clinical practice, policy experts and, ultimately, recipients of care will all depend upon the integrity of the publication process to objectively select and efficiently distribute the useful information from the recommended publications in order to serve as the basis for practice guidelines and standards, as well as continuous development of nursing science. ${ }^{4,5}$ In order to communicate effectively with their readers, the published articles have to be user friendly - conveying a clear and precise message in a common language, and an easy-to-read format.

As scientists at the gate ${ }^{6}$ peer reviewers are asked to read assigned manuscripts and write reviews that communicate their best judgment about the originality, significance and validity of the research articles. In my past decade of experiences of being a peer reviewer of international nursing and health care journals, I have had to scrutinize the submitted papers and fully understand the meaning of every sentence, positioning myself as the future reader of the article. The succinctness and clarity of the writing style of the submission should also be evaluated. ${ }^{7}$ Therefore, contrary to what 
Beckstead $^{2}$ suggested, while peer reviewers should read and comment on the manuscript, they should also be fully aware that the purpose of most peer-reviewed scientific nursing journals is to communicate effectively to nurse and health care researchers, practitioners and administrators, whose formal education may have been at different levels about the main findings of the studies and their implications for education and practice, as well as their scholarly views on literature and policies. In addition, a few journals whose aim is to disseminate advances in nursing sciences and specialized areas of knowledge, such as Advances in Nursing Sciences and Nursing and Health Sciences, may require specific and advanced knowledge and readership in nursing philosophy, theory and specialty.

\section{Peer review system and its monitoring}

An effective peer review system consists of the process of unbiased, independent and critical assessment of manuscripts submitted to journals by experts who are not part of the editorial staff. The system or process of peer review is an intrinsic part of all scholarly work, including the scientific process, as well as the comprehensiveness, constructiveness and clarity of the reviewers' reports and comments that would certainly affect final decision on the disposal (i.e., acceptance, revision required or rejection) of the submitted manuscripts made by the responsible editors. In this paper, I would like to highlight three major concerns to be addressed in peer review system of scientific nursing journals so as to arouse more attention and discussion about this topic. First, most of the scientific journals have only provided a brief outline and general guidance for review in written form and novice reviewers would often find the reviewing work being very difficult, over demanding, confused, and thus frustrated. Those novice reviewers should have been provided with adequate training or supervised practices of manuscript review (i.e. at least checking whether they are comfortable with starting an review independently) before they can better understand the specific requirements of the journal on the manuscript writing and review technique. However, many journals have only provided with online tutorials of review for reviewers and thus whether the novice reviewers (and even the experienced ones) can give clear, concise and constructive comments to the authors and/or help the editor make a decision on the acceptance of the manuscript would be questionable.

Second, editors of the scientific journals have to assign appropriate expert reviewers to comment on submitted manuscripts and select the best written articles for publication using comments from more than one reviewer and the responses to reviewers' comments from authors. Preparation of a succinct and fair evaluation by a reviewer is essential, not only for generating high quality articles in scientific nursing journals, but also for contributing to scholarly dialogues between clinical, research, theoretical, or philosophical experts (authors and reviewers). However, International Committee of Medical Journal Editors'3 query about whether there are sufficient reviewers with relevant expertise on the topic of the manuscript for the editor to select in the peer review process. As no attractive reward or apparent benefit obtained by the reviewers except contributions to scientific development and evidencebased practice in nursing, they often have to their own free time and effort for such review work. Therefore from time to time, it is very much difficult for the editors to identify appropriate reviewers for the submitted manuscripts with wide varieties of research topics or areas. Hence, the Committee's comments that there is a need for evaluation of the feasibility and sustainability of the peer review system of a particular journal, especially when the number of peer reviewers is not adequate for implementing an efficient and satisfactory quality peer review on different submitted manuscripts with a wide variety of research topics or methods. Last but not least, most of the scientific nursing journals do not have a structured and regular monitoring system of the quality of peer review. Throughout the review process, reviewers have to integrate their knowledge and experience in health care services and research with their critical analysis, imagination and innovation during the peer review process. They are then required to write up their comments on the manuscripts in a clear, logical and well-organized manner so that authors are able to improve both the report writing skills and integrity and clarity of the research process. The merits of a peer review can be revealed from the structure and content of the written comments, which follow the guide for reviewers provided by the journal and would certainly also refer to the critical thinking ability and process of the reviewer. High quality reviews should be presented in a fair, constructive, precise and focused manner that facilitates communication of key messages about the standard and importance of the manuscript for publication. ${ }^{8}$ There should be a need for a standardized control system of the quality of peer review formulated by individual journals to regularly assess on the peer review process, particularly the structure and content of reviewer's narrative comments, duration of response to invitation for and completion of review, and inter-reviewer agreement on recommendation and grading of the manuscript.

\section{Quality improvement of peer review in scientific journals}

The editors of peer-reviewed scientific journals have the responsibility of monitoring the quality of the peer review process. However, appraising the quality of peer review is not an easy task. Evaluation of the quality of written comments by the reviewers can be a highly subjective judgment; however, inter-rater agreement is crucial to a more accurate interpretation of quality scores for narrative comments in peer reviews. Medical editors (e.g., Obstetrics and Gynecology) reach acceptable levels of inter-rater agreement and reliability for review quality scores using a standardized assessment tool such as Review Quality Index, and the intra-class correlation statistic. ${ }^{9,10}$ An easy-to-use, standardized assessment protocol, that produces reliable and accurate scores, is useful and important when monitoring quality, in peer review. The top-ranked nursing journals such as Nursing Research adopted a continuous improvement framework to develop a methodology for assessing the quality of the narrative component of peer reviews of submitted manuscripts, using a standardized assessment instrument called the General Assessment of the Reviews of Nursing Research (GARNER). The core dimension of the review quality is the adequacy of the argument used in support of a judgment about the significance of a scientific manuscript ${ }^{11}$ and the usefulness of narrative comments is assessed from eight aspects, including construction, knowledge, balance or impartiality, logic, clarity, precision, usefulness to authors, and usefulness to the editor. There is no perfectly accurate and reliable tool for quality control but a simple system or measuring tool for periodically grading review quality, should be in place. As such, the regular monitoring system can suggest room for improvement in the peer review. $^{12}$

Apart from a valid tool for monitoring the quality of reviews, editors usually use clear guidelines for reviewers in order to spell out their expectations or the journal's requirement for reviewers' comments to the authors (i.e. highlighting the focused areas or aspects to be commented on). However, not every scientific journal has a clear and detailed guideline to inform reviewers about the desired format, or the elements or statements to be used in evaluating different kinds of manuscripts (e.g., writing a comment with explanation and examples to illustrate the underlying reasons for the suggestion made). The reviewers may have problems to prepare thorough and valid comments on the manuscript for the authors. New reviewers may also find great difficulty in understanding the peer review process and the expectations in terms of format and content of a clear and constructive review. A few examples of review practice, supervised by an experienced reviewer if possible, can be set up for the better orientation of novice reviewers so that their reports can be compared with those made by the experienced reviewer and written 
feedback can also be evaluated by an editorial board member

We understand that peer review in scientific nursing journals is a voluntary work and important contributions to the dissemination of research evidence and professionalization in nursing. With continuous improvement in the quality of peer review, reviewers, authors, and editors will enjoy and learn from the review process, while the readers and health care consumers will benefit from the publication of research reports and systematic reviews of the highest standard and significance to nursing science and practice. I hope the discussion in this paper can encourage careful consideration by editorial panels and stimulate further discussion among these panel members, existing and potential reviewers and researchers about the peer review system of nursing journals and its quality control, which are integral parts of a high impacted scientific journal.

\section{References}

1. Van Rooyen S, Godlee F, Evans S, et al. Effect of open peer review on quality of reviews and on reviewers' recommendations: a randomised trial. BMJ 1999;318: 23-7.

2. Beckstead JW. Peer-reviewed scientific journals in nursing: to whom are we writing? Int J Nurs Stud 2009;46;1166-7.

3. International Committee of Medical Journal Editors. Ethical Considerations in the Conduct and Reporting of Research: Peer Review. c2009. Available from: http://www.icmje.org/ethical_3peer.html

4. Callaham ML, Knopp RK, Gallagher EJ. Effect of written feedback by editors on quality of reviewers. JAMA 2002;287:27813.

5. Walker K. 'Double b(l)ind': peer-review and the politics of scholarship. Nurs Phil 2004;5:135-46.

6. Drazen JM. Scientists at the gate [Letter].
New York Times 2006 May 9.

7 Godlee F, Jefferson T. Peer reviewer in health sciences. 2nd ed. London: BMJ Books; 2003.

8. Bearinger LH. Beyond objective and balanced: writing constructive manuscript reviews. Res Nurs Health 2006;29:71-3.

9. Black N, van Rooyen S, Godlee F, et al. What makes a good reviewer and a good review in a general medical journal? JAMA 1998;280:231-3.

10. Landkroon AP, Euser AM, Veeken H, et al. Quality assessment of reviewers' report using a simple instrument. Obstet Gynecol 2006;108:979-85.

11. Hames I. Peer review and manuscript management in scientific journals. Guidelines for good practice. Oxford, UK: Blackwell; 2007.

12. Freda MC, Kearney MH, Baggs JG, et al. Peer reviewer training and editor support: results from an international survey of nursing peer reviewers. J Prof Nurs 2009; 25:101-8. 